\title{
Uncertainty Quantification of Charge Transfer through a Nanowire Resonant-Tunneling Diode with an ADHIE-FDTD method
}

\author{
Pieter Decleer \\ Quest / Dept. of Information Technology \\ Ghent University / imec \\ Gent, Belgium \\ Email: pieter.decleer@ugent.be
}

\begin{abstract}
The influence of barrier thickness variability on the charge transfer characteristics of an InP/InAs/InP nanowire resonant-tunneling diode is studied. The transmission probability through the diode is calculated by solving the timedependent effective-mass Schrödinger equation with the Alternating-Direction Hybrid Implicit-Explicit (ADHIE) FiniteDifference Time-Domain (FDTD) method. This recently developed method is tailored towards multiscale problems and thus allows for a much faster evaluation of the transmission probability compared to the commonly used leapfrog FDTD method. Accurate and efficient modeling of small geometric features with the ADHIE-FDTD method now facilitates the development of a robust Monte Carlo method to assess the significant influence of the thickness of the barriers on the transmission probability and the current-voltage characteristic.
\end{abstract}

Keywords - Nanowire Resonant-Tunneling Diode, FiniteDifference Time-Domain, Uncertainty Quantification.

\section{INTRODUCTION}

Electronic devices are being scaled down to the nanoscale. As part of their design, they are using components such as quantum wires [1-4] and quantum waveguides [5-7] that rely on quantum confinement. To better understand, model and design these devices, it is not sufficient that analytical solutions are found for relatively easy geometries. Instead, it is imperative that we develop numerical techniques able to efficiently simulate the behavior of more complex and multiscale nanodevices.

Charge transport through these components can be described with the time-dependent Schrödinger equation. This equation - among many others - is regularly solved by adopting a Finite-Difference Time-Domain (FDTD) method [8-13] which iterates the wave function from one point in time to the next. However, this method is hindered by severe restrictions on the time step between iterations when applied to highly multiscale quantum devices. To alleviate these restrictions, an improvement was recently developed: the Alternating-Direction Hybrid ImplicitExplicit (ADHIE) FDTD method [14, 15].

Another challenge is related to uncertainty introduced into these devices due to imperfect production processes or variable operating conditions [16]. To ensure the proper working of a device, it is crucial to assess how strongly manufacturing variability influences its behavior. This uncertainty quantification (UQ) can be done by constructing a stochastic model. One such approach is to use a brute-force

\author{
Dries Vande Ginste \\ Quest / Dept. of Information Technology \\ Ghent University / imec \\ Gent, Belgium
}

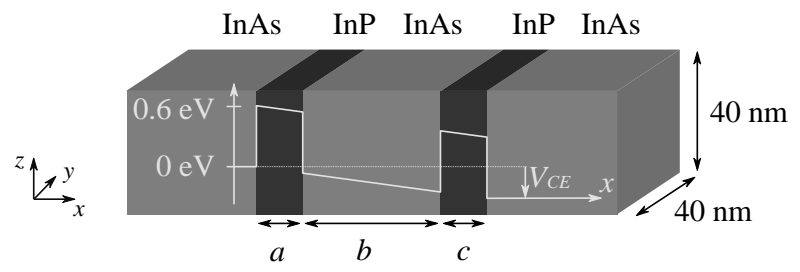

Fig. 1. Geometry of the InP/InAs/InP nanowire resonant-tunneling diode. The nanowire is assumed infinite in the $x$-direction and has a square cross-section with equal sides of $40 \mathrm{~nm}$. A voltage $V_{C E}$ is applied across the double barrier structure, resulting in a linear decresase of the potential energy surface. The two InP barriers have a height of $0.6 \mathrm{eV}$ and a width $a$ and $c$ for the left and right barrier, respectively. The middle InAs region has a width $b$. The effective mass of an electron in InAs and InP are $m_{\mathrm{InAs}}=0.023 \mathrm{~m}_{\mathrm{e}}$ and $m_{\mathrm{InP}}=0.077 \mathrm{~m}_{\mathrm{e}}$, respectively.

Monte Carlo (MC) method [17], which is essentially a sampling technique. It is a popular method as it is robust, reliable and easy to implement. Unfortunately, it requires a large number of samples, thus necessitating very efficient methods to obtain these samples.

In this work, the effect of variability in the barrier thickness of an InP/InAs/InP nanowire resonant-tunneling diode (RTD) is studied. Thereto, the time-dependent effective-mass Schrödinger equation is solved using the ADHIE-FDTD method. From this time-domain simulation, the transmission probability as a function of energy for a specific applied voltage is obtained. From the transmission probability, the IV-characteristic of the device is determined. Next, an MC method is implemented to build a stochastic model of the nanowire RTD. As will be shown, thanks to the much increased efficiency of ADHIE-FDTD compared to leapfrog FDTD, it becomes feasible to use the MC approach and reveal the critical influence of the variability on the RTD's operation.

\section{AN INP/INAS/INP NANOWIRE RESONANT-TUNNELING DIODE}

The geometry of the InP/InAs/InP nanowire resonanttunneling diode (RTD) under study is shown in Fig. 1. This structure is similar to the one discussed in [18, 19] and constructed in [3]. The nanowire has a square cross-section with a $40 \mathrm{~nm}$ side length and is assumed infinite in the $x$ direction. The RTD is defined by two InP barriers of height $V=0.6 \mathrm{eV}$. The effective masses in InAs and InP are $m_{\text {InAs }}=0.023 \mathrm{~m}_{\mathrm{e}}$ and $m_{\mathrm{InP}}=0.077 \mathrm{~m}_{\mathrm{e}}$, respectively. Due to the 
fabrication process there is an inherent uncertainty in the width of the barriers. Therefore, the width $a$ of the left barrier and the width $c$ of the right barrier are modeled as correlated random variables (RVs). The middle region's width is given by $b=20 \mathrm{~nm}-(a+c) / 2$.

In $[18,19]$ it is argued - based on a self-consistent 3-D Poisson-Schrödinger calculation - that the "top hat" potential profile, illustrated in Fig. 1, is a very good approximation to the true potential profile. It was also shown that only the lowest transversal eigenstate contributes to the conduction and that the Fermi-level is only $2 \mathrm{meV}$ above this lowest 1-D sub-band. These results were in correspondence with the experimental results in [3]. In this work, the lowest transversal eigenstate corresponds to a particle-in-a-2-D-box with ground state energy:

$$
E_{0}=20.436 \mathrm{meV} \text {. }
$$

\section{NUMERICAL METHOD}

The transmission probability and the corresponding IVcharacteristic of the device is calculated by solving the timedependent effective-mass Schrödinger equation:

$$
\jmath \hbar \frac{\partial \psi}{\partial t}=-\frac{\hbar^{2}}{2} \nabla \cdot\left(\frac{1}{m} \nabla \psi\right)+V \psi,
$$

Here, $J$ is the imaginary unit, $\hbar$ the reduced Planck's constant, $\psi$ the wave function, $m$ the effective mass and $V$ the background potential.

\section{A. The Alternating-Direction Hybrid Implicit-Explicit Finite-Difference Time-Domain Method}

To solve the Schrödinger equation, the recently developed ADHIE-FDTD method is used [14]. For a mass varying along a single direction (here, the $x$-direction), this method discretizes the wave function on a rectilinear grid containing $N=n_{x} \times n_{y} \times n_{z}$ cells and propagates it from time $t=n \Delta t$ to time $t=(n+1) \Delta t$ by solving the following discretized linear system:

$$
\left.\left[\begin{array}{cc}
G & -\Delta v H \\
0 & G
\end{array}\right] \boldsymbol{x}\right|^{n+1}=\left.\left[\begin{array}{cc}
G & 0 \\
-\Delta v H & G
\end{array}\right] \boldsymbol{x}\right|^{n} .
$$

The vector $\left.\boldsymbol{x}\right|^{n+1}$ stacks the row-major vectorized real and imaginary parts of the wave function at time steps $t=(n+1) \Delta t \quad$ and $t=(n+1 / 2) \Delta t, \quad$ respectively. The normalized time-step $\Delta v$ is given by $\Delta v=\Delta t / \hbar$. The matrices $G$ and $H$ are defined as:

$$
\begin{gathered}
G=\left(I_{n_{x}-1}+\frac{\Delta v}{2} S_{x}\right) \otimes I_{n_{y}-1} \otimes I_{n_{z}-1}, \\
H=T_{x}+T_{y}+T_{z}+V,
\end{gathered}
$$

where $I_{n}$ is the unit matrix of size $n, \bigotimes$ is the Kronecker product, $V$ contains the potential surface and the matrices $S_{x}$, $T_{x}, T_{y}$, and $T_{z}$ are given by:

$$
\begin{gathered}
S_{x}=\frac{\hbar^{2}}{2}\left(\delta_{x}^{*-1} D_{x}^{T} m^{*-1} P_{x} \delta_{x}^{-1} D_{x}\right), \\
T_{x}=\frac{\hbar^{2}}{2}\left(\delta_{x}^{*-1} D_{x}^{T} m^{*-1} \delta_{x}^{-1} D_{x}\right) \otimes I_{n_{y}-1} \otimes I_{n_{z}-1}, \\
T_{y}=\frac{\hbar^{2}}{2} m^{-1} \otimes\left(\delta_{y}^{*-1} D_{y}^{T} \delta_{y}{ }^{-1} D_{y}\right) \otimes I_{n_{z}-1},
\end{gathered}
$$

$$
T_{z}=\frac{\hbar^{2}}{2} m^{-1} \otimes I_{n_{y}-1} \otimes\left(\delta_{y}^{*-1} D_{y}^{T} \delta_{y}^{-1} D_{y}\right) .
$$

The diagonal matrices $\delta_{x}$ and $\delta_{x}{ }^{*}$ contain the $n_{x}$ primary and $n_{x}-1$ dual grid steps in the $x$-direction, and similarly for $y$ and $z$. The discrete differentiator $D_{x}$ is defined as:

$$
D_{x}=\left[\begin{array}{cccc}
-1 & & & \\
1 & -1 & & \\
& \ddots & \ddots & \\
& & 1 & -1 \\
& & & 1
\end{array}\right]_{n_{x} \times\left(n_{x}-1\right)},
$$

and similarly for the $y$ - and $z$-directions. The diagonal matrix $m$ contains the variable mass in the $x$-direction on the vertices of the grid, excluding the edge of the grid. The matrix $m^{*}$ contains the harmonically averaged mass.

The strength of the novel method stems from the diagonal matrix $P_{x}$ which determines the method's HIE character [14]. While the traditional leapfrog FDTD method [12, 20] is fast, easy to implement and memory efficient, it is also hindered by a strict condition on the time step. The maximum allowed time step is determined by the minimum cell size. In highly multiscale set-ups where fine geometric details have to resolved locally, this limit becomes detrimental to the efficiency. In contrast, the ADHIE-FDTD method can exclude small cells from the stability criterion at will. In particular, the diagonal projection matrix $P_{x}$ contains a 1 instead of a 0 at position $i$ if $\Delta x_{i}$ should be excluded from the stability criterion, and thus treated implicitly. This increases the cost per iteration only slightly while increasing the overall efficiency dramatically. In this work, we fully exploit this property: to accurately resolve the wave phenomena at and between the barriers, we discretize them finely but solve them implicitly. As such, the efficiency is not compromised.

A wave packet is inserted into the computational domain by using a Total-Field Scattered-Field (TFSF) boundary [7] and the computational domain is terminated by Perfectly Matched Layers (PML) [21-23].

\section{B. Transmission probability and current}

The transmission probability $T$ of a wave packet through the double barrier structure is dependent on both the incoming energy $E$ of the wave packet and the applied voltage $V_{C E}$. It is determined by calculating the average of the outgoing wave function over the transversal direction, taking the Fourier transform of this signal and dividing it by the analytical signal when no barrier or bias is present:

$$
T\left(E, V_{C E}\right)=\left|\frac{\Psi_{\text {calc }}\left(E, V_{C E}\right)}{\Psi_{\text {ana }}(E)}\right|^{2} .
$$

To resolve the extremely narrow transmission peaks, the time-domain signal is extrapolated using the matrix-pencil method [24]. The current is calculated from the transmission probability as in [25], by taking Fermi-Dirac statistics for the occupation levels at the terminals and the 1-D density of states into account: 


$$
\begin{aligned}
I\left(V_{C E}\right) & =\frac{2 e}{\pi \hbar} \sqrt{E_{0}} \int_{E_{0}}^{\infty} T\left(E, V_{C E}\right)\left(E-E_{0}\right)^{-\frac{1}{2}} \times \\
& \left(\frac{1}{1+\exp \left(\frac{E-E_{F}}{k_{B} T}\right)}-\frac{1}{1+\exp \left(\frac{E-E_{F}-e V_{C E}}{k_{B} T}\right)}\right) \mathrm{d} E,
\end{aligned}
$$

where $e$ is the electron charge, $k_{B}$ is the Boltzmann constant and $T$ is the temperature, chosen to be $4.2 \mathrm{~K}$ as in $[3,18,19,25]$.

To calculate the IV-characteristic via (12), the Schrödinger equation (2) is solved by means of the ADHIEFDTD method for every applied voltage $V_{C E}$.

\section{VARIABILITY ANALYSIS}

The calculation of the transmission (11) over a broad range of applied voltages $V_{C E}$ and for many variations of the barrier widths $a$ and $c$ would not be possible with the traditional leapfrog FDTD method due to the multiscale device dimensions and the consequent small time steps. The ADHIE-FDTD method now allows faster evaluation of the transmission and facilitates the variability analysis of the influence of barrier thickness by means of stochastic modeling. This UQ is implemented using the MC method.

\section{A. Dimensioning of the barriers and simulation set-up.}

Consider the nanowire RTD in Fig. 1. An MC run is performed for 4000 samples of the RVs $a$ and $c$. The RVs were picked from a bivariate normal distribution with means $\mu_{a}=\mu_{c}=5.0 \mathrm{~nm}$ and standard deviations $\sigma_{a}=\sigma_{c}=0.1 \mathrm{~nm}$ with a correlation coefficient $\rho=0.8$. The rather high correlation coefficient reflects that uncertainties in the production process result in similar deviations from the nominal value for both barriers. Note that the UQ method presented in this work is in no way restricted to this specific probability distribution of $a$ and $c$. Other distributions, e.g., acquired by measuring the physical dimensions of many manufactured RTDs, can be dealt with too.

The device is discretized in the $x$-direction from $-200 \mathrm{~nm}$ to $200 \mathrm{~nm}$ with the active region of the device centered at $0 \mathrm{~nm}$. From $-200 \mathrm{~nm}$ to $-30 \mathrm{~nm}$ and from $30 \mathrm{~nm}$ to $200 \mathrm{~nm}$ the grid has relatively large, uniform cells of width $\Delta x=2.0 \mathrm{~nm}$. The narrow InP barriers, however, are very finely discretized with 20 cells each and the intermediate InAs region is discretized with 60 cells. The cells from $-30 \mathrm{~nm}$ to the left side of the first barrier are scaled with a constant grading ratio and similarly for the right side of the device. The computational domain is terminated by a $150 \mathrm{~nm}$ thick PML on both sides. The $y$ - and $z$-directions are discretized with 20 cells of width $\Delta x=2.0 \mathrm{~nm}$. A Gaussian wave packet is inserted into the computational domain with central energy $E=0.08 \mathrm{eV}$ and a width of $\sigma=10 \mathrm{~nm}$ using a TFSF boundary.

The cells in the $x$-direction between $-30 \mathrm{~nm}$ and $30 \mathrm{~nm}$ are solved implicitly while the other parts are solved explicitly. As such, the spatial refinement around the active region of the device does not influence the maximum allowed time step. The resulting time step is $\Delta t=256$ as. If instead no implicitization was applied, as in leapfrog FDTD, the maximum time step would be $\Delta t=12$ as, rendering an $\mathrm{MC}$ analysis intractable. Thanks to the HIE approach, however,

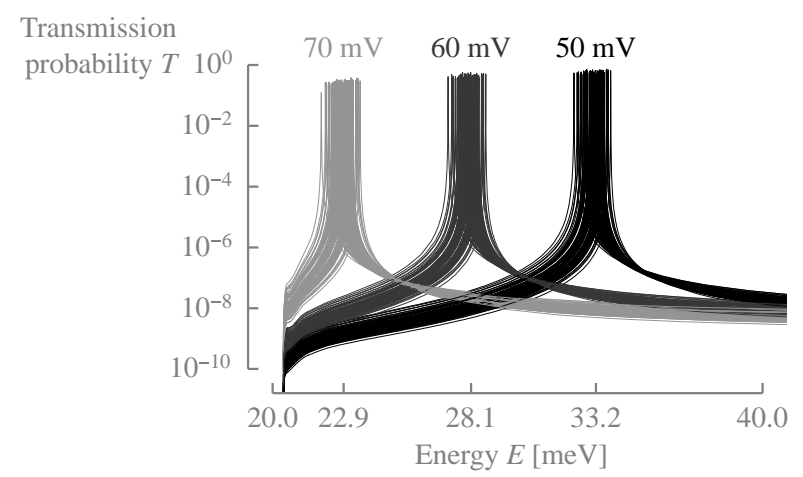

Fig. 2. The position of the transmission peaks exhibits a large variability. Specifically, the transmission probability $T$ as function of energy $E$ for $V_{C E}$ at $50 \mathrm{mV}, 60 \mathrm{mV}$ and $70 \mathrm{mV}$ for 100 out of the 4000 MC samples is shown. The tick marks on the abcissa represent the mean peak position $\mu_{\mathrm{T}}$ for the different applied voltages. A change in applied voltage of approximately $10 \mathrm{mV}$ results in a shift in the transmission peak of approximately $5 \mathrm{meV}$.

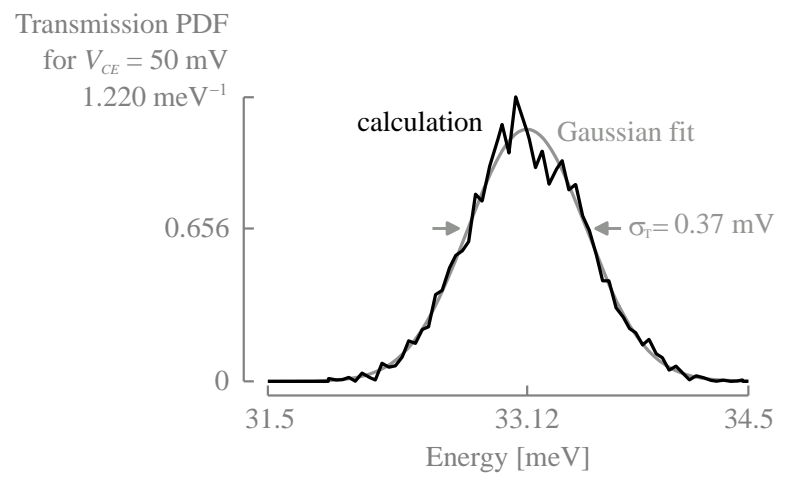

Fig. 3. The position of the transmission peak as a function of energy has a Gaussian PDF for an applied voltage of $V_{C E}=50 \mathrm{meV}$. The mean position for the transmission peak is $\mu_{\mathrm{T}}=33.12 \mathrm{meV}$ with a standard deviation of $\sigma_{\mathrm{T}}=0.37 \mathrm{meV}$. The fitted curve is a Gaussian with the same mean and standard deviation. A similar graph is obtained for other applied voltages.

the single-core time to perform one ADHIE-FDTD simulation on a laptop with an Intel® ${ }^{\circledR}$ Core $^{\text {TM }}$ i7-8650U CPU @ $1.90 \mathrm{GHz}$ with $16.0 \mathrm{~GB}$ of RAM is $167 \mathrm{~s}$.

\section{B. Results}

The transmission probability as a function of the energy for 100 out of the 4000 samples is shown in Fig. 2 for three different applied voltages $V_{C E}=50 \mathrm{mV}, 60 \mathrm{mV}$ and $70 \mathrm{mV}$. It is seen that the transmission exhibits very fine peaks due to a very sharp resonance and that the position of these peaks is very sensitive to the dimensions of the barriers. The probability density function (PDF) of the position of the peak is shown in Fig. 3 for an applied voltage of $V_{C E}=50 \mathrm{mV}$. The distribution is approximately Gaussian with a mean of $\mu_{\mathrm{T}}=33.12 \mathrm{meV}$ and standard deviation $\sigma_{\mathrm{T}}=0.37 \mathrm{meV}$. Increasing the applied voltage by $1 \mathrm{mV}$ shifts the mean value of the peak by approximately $0.5 \mathrm{meV}$. This implies that the $\mu_{\mathrm{T}} \pm \sigma_{\mathrm{T}}$ regions for two applied voltages differing by $1 \mathrm{mV}$ partially overlap. Consequently, the device cannot be expected to reliably operate within $1 \mathrm{mV}$ of accuracy.

To assess the convergence of the MC analysis, the relative error of the mean $\mu_{\mathrm{T}}$ and standard deviation $\sigma_{\mathrm{T}}$, for an applied voltage of $50 \mathrm{mV}$, is shown in Fig. 4 as a function of $N$, i.e., the number of MC samples. The expected decrease of the 


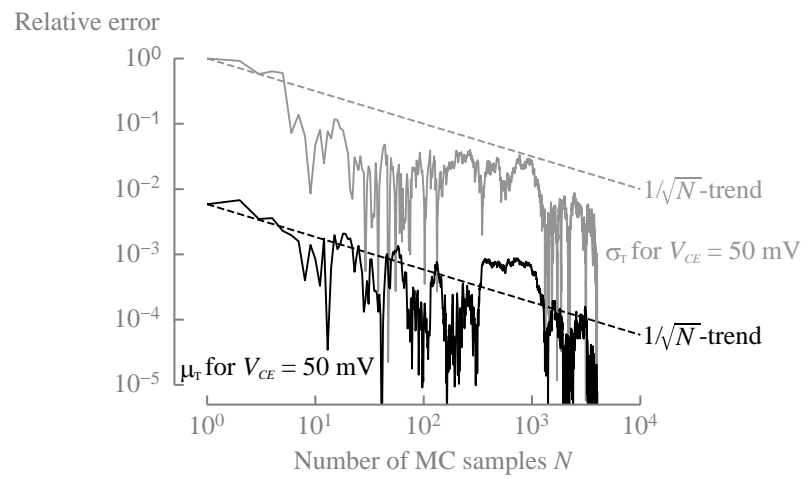

Fig. 4. The relative error of the mean $\mu_{\mathrm{T}}$ and standard deviaton $\sigma_{\mathrm{T}}$ of the position of the transmission peak decrease as the square root of the number of Monte Carlo samples $N$. This is illustrated for an applied voltage of $V_{C E}=50 \mathrm{mV}$. The behavior for other applied voltages is similar.

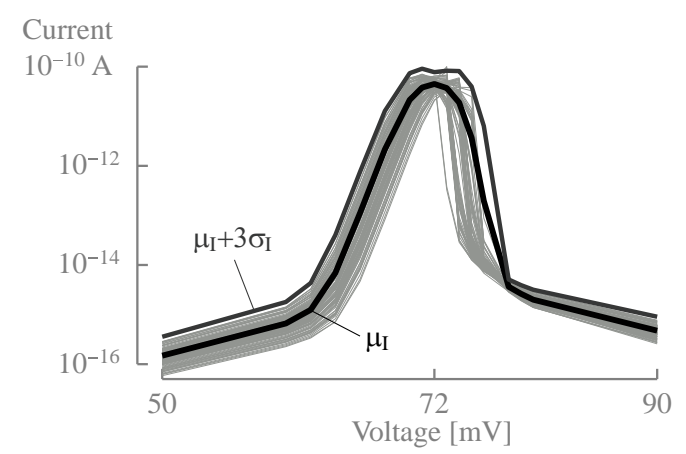

Fig. 5. The current-voltage curves of 100 out of 4000 MC samples clearly show a region of negative differential resistance, characteristic of a resonant-tunneling diode. The thick black line indicates the mean $\mu_{\mathrm{I}}$ and the thinner black line indicates the $\mu_{I}+3 \sigma_{I}$ boundary, where $\sigma_{I}$ is the standard deviation. The mean has a maximum current at $72 \mathrm{mV}$. There is a large variation in the position of the peak current and the region of negative differential resistance.

error as $1 / \sqrt{N}$ is clearly observed. Note that many samples, here 4000 , are needed to have an error of less than $1 \%$ on the standard deviation.

The resulting current-voltage characteristics of the device for 100 samples are shown in Fig. 5. The mean IV-curve

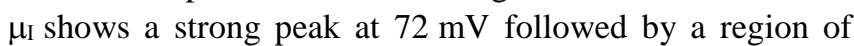
negative differential resistance, characteristic of an RTD. It is also seen that there is a large variance in the position of the current peak and the region of negative differential resistance. These are two very important quantities for obtaining a correctly functioning device. As such, it is crucial that we assess how much fabrication uncertainties affect device operation. Moreover, there is also a large variance in the amplitude of the current at any given voltage $V_{C E}$. In Fig. 6 , the PDF for the current at applied voltages of (a) $50 \mathrm{mV}$ and (b) $70 \mathrm{mV}$ are shown. Both distributions are very skew, with rather long tails towards high currents. For example, in $10 \%$ of the cases, the current for an applied voltage of $70 \mathrm{mV}$ will exceed approximately triple the mean value of the current. Hence it is clear that, instead of merely estimating the mean and standard deviation, the full quantification of the statistics, including the distribution's tail, is essential for a design engineer. It allows to assess in how many cases, the device will carry too much current, possibly leading to failure of

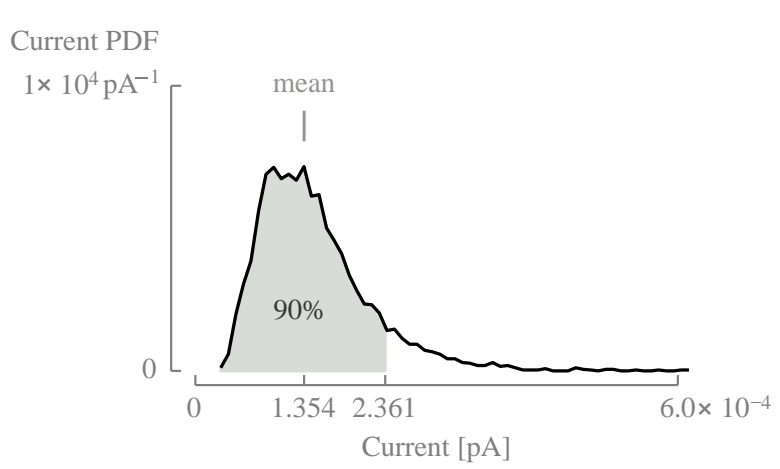

(a) $V_{C E}=50 \mathrm{mV}$

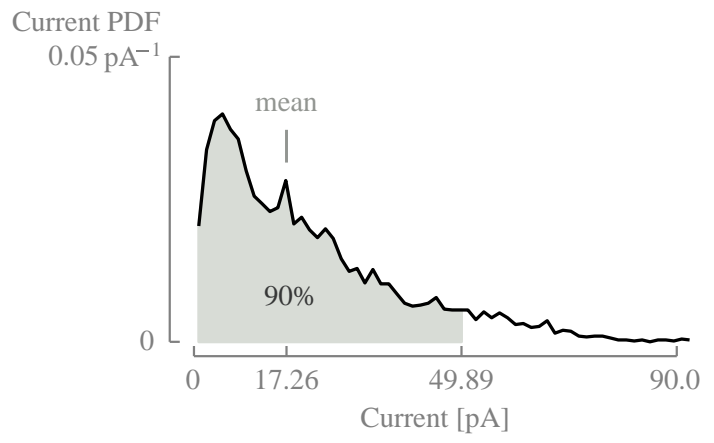

(b) $V_{C E}=70 \mathrm{mV}$

Fig. 6. The probability density functions show that the current exhibits a large variance. This is illustrated for an applied voltage $V_{C E}$ of $50 \mathrm{mV}$ in (a) and $70 \mathrm{mV}$ in (b). In both cases the distributions are very skew with a long tail towards high currents. In $10 \%$ of the cases the current will exceed almost double the mean current for (a) and almost triple the mean current for (b).

other components. As such, the advocated approach may be used for yield analysis.

\section{CONCLUSION}

In this work, uncertainty quantification of an InP/InAs/InP nanowire resonant-tunneling diode was performed leveraging an ADHIE-FDTD method for the numerical solution of the pertaining Schrödinger equation. Specifically, a stochastic model was built to investigate the influence of the barrier width on charge transport with a Monte Carlo approach. It was shown that, thanks to the ADHIE-FDTD method, this kind of UQ becomes tractable, paving the way for performing variability analysis on more advanced nanoscale devices. Moreover, it was demonstrated that, to assess the robustness of a design, it is crucial to have knowledge of the full statistics of the device's behavior, described, e.g., by means of the PDF of critical output parameters.

\section{REFERENCES}

[1] K. Tomioka, M. Yoshimura, and T. Fukui, "A III-V nanowire channel on silicon for high-performance vertical transistors," Nature, vol. 488, no. 7410, pp. 189-192, Aug. 2012.

[2] M. Sistani et al., "Room-Temperature Quantum Ballistic Transport in Monolithic Ultrascaled Al-Ge-Al Nanowire Heterostructures," Nano Lett., vol. 17, no. 8, pp. 4556-4561, Aug. 2017.

[3] M. T. Björk et al., "Nanowire resonant tunneling diodes," Appl. Phys. Lett., vol. 81, no. 23, pp. 4458-4460, 2002.

[4] B.-H. Lee et al., "A Vertically Integrated Junctionless Nanowire Transistor,” Nano Lett., vol. 16, no. 3, pp. 1840-1847, Mar. 2016. 
[5] J. A. del Alamo and C. C. Eugster, "Quantum field - effect directional coupler," Appl. Phys. Lett., vol. 56, no. 1, pp. 78-80, Jan. 1990.

[6] M. Yamamoto, S. Takada, C. Bäuerle, K. Watanabe, A. D. Wieck, and S. Tarucha, "Electrical control of a solid-state flying qubit," Nat. Nanotechnol., vol. 7, no. 4, pp. 247-251, 2012.

[7] J. F. Mennemann and A. Jüngel, "Perfectly Matched Layers versus discrete transparent boundary conditions in quantum device simulations," J. Comput. Phys., vol. 275, pp. 1-24, 2014.

[8] J. Shen, W. E. I. Sha, Z. Huang, M. Chen, and X. Wu, "High-order symplectic FDTD scheme for solving a time-dependent Schrödinger equation," Comput. Phys. Commun., vol. 184, no. 3, pp. 480-492, 2013.

[9] W. C. Tay and E. L. Tan, "Pentadiagonal alternating-direction-implicit finite-difference time-domain method for two-dimensional Schrödinger equation," Comput. Phys. Commun., vol. 185, no. 7, pp. 1886-1892, 2014.

[10] J. P. Wilson, "Generalized Finite-Difference Time-Domain method with absorbing boundary conditions for solving the nonlinear Schrödinger equation on a GPU," Comput. Phys. Commun., vol. 235, pp. 279-292, 2019.

[11] A. Askar and A. S. Cakmak, "Explicit integration method for the timedependent Schrodinger equation for collision problems," J. Chem. Phys., vol. 68, no. 6, pp. 2794-2798, 1978.

[12] P. Decleer, A. Van Londersele, H. Rogier, and D. Vande Ginste, "Nonuniform and Higher-order FDTD Methods for the Schrödinger Equation," J. Comput. Appl. Math., vol. 381, p. 113023, Jan. 2021.

[13] W. C. Tay and E. L. Tan, "Pentadiagonal alternating-direction-implicit finite-difference time-domain method for two-dimensional Schrödinger equation," Comput. Phys. Commun., vol. 185, no. 7, pp. 1886-1892, 2014.

[14] P. Decleer, A. Van Londersele, H. Rogier, and D. Vande Ginste, "An alternating-direction hybrid implicit-explicit finite-difference timedomain method for the Schrödinger equation," unpublished.

[15] A. Van Londersele, D. De Zutter, and D. Vande Ginste, "An in-depth stability analysis of nonuniform FDTD combined with novel local implicitization techniques,” J. Comput. Phys., vol. 342, pp. 177-193, 2017.

[16] P. Krantz, M. Kjaergaard, F. Yan, T. P. Orlando, S. Gustavsson, and W. D. Oliver, "A quantum engineer's guide to superconducting qubits," Appl. Phys. Rev., vol. 6, no. 2, p. 021318, Jun. 2019.

[17] M. H. Kalos and P. A. Whitlock, Monte Carlo Methods. Whiley-VCH, 2009.

[18] M. Zervos and L. F. Feiner, "Electronic structure of piezoelectric double-barrier InAs/InP/InAs/InP/InAs (111) nanowires," J. Appl. Phys., vol. 95, no. 1, pp. 281-291, 2004.

[19] M. Zervos and N. T. Pelekanos, "Current transport in semiconductor nanowires with built-in barriers based on a 1D transfer matrix calculation," J. Appl. Phys., vol. 104, no. 5, 2008.

[20] P. B. Visscher, "A fast explicit algorithm for the time-dependent Schrödinger equation." Comput. Phys., vol. 5, no. 6, p. 596, 1992.

[21] D. M. Sullivan and P. M. Wilson, "Time-domain determination of transmission in quantum nanostructures," J. Appl. Phys., vol. 112, no. 6, p. 064325, 2012.

[22] J. F. Mennemann and A. Jüngel, "Perfectly Matched Layers versus discrete transparent boundary conditions in quantum device simulations," J. Comput. Phys., vol. 275, pp. 1-24, 2014.

[23] C. Zheng, "A perfectly matched layer approach to the nonlinear Schrödinger wave equations," J. Comput. Phys., vol. 227, no. 1, pp. 537-556, 2007.

[24] J. Ritter, and F. Arndt, "Efficient FDTD/Matrix-pencil method for the full-wave scattering parameter analysis of waveguiding structures," IEEE Trans. Microw. Theory Tech., vol. 44, no. 12 PART 2, pp. 24502456, 1996

[25] R. Ragi, R. V. T. da Nobrega, and M. A. Romero, "Modeling of peak voltage and current of nanowire resonant tunneling devices: case study on InAs/InP double-barrier heterostructures," Int. J. Numer. Model. Electron. Networks, Devices Fields, vol. 26, no. 5, pp. 506-517, Sep. 2013. 